\title{
MODELING SARS-CoV-2 SPREAD WITH DYNAMIC ISOLATION
}

\author{
MD. AZMIR IBNE ISLAM, SHARMIN SULTANA SHANTA, AND ASHRAFUR RAHMAN
}

\begin{abstract}
Background: The SARS-CoV-2 pandemic is spreading with a greater intensity across the globe. The synchrony of public health interventions and epidemic waves signify the importance of evaluation of the underline interventions.
\end{abstract}

Method: We developed a mathematical model to present the transmission dynamics of SARSCoV-2 and to analyze the impact of key nonpharmaceutical interventions such as isolation and screening program on the disease outcomes to the people of New Jersey, USA. We introduced a dynamic isolation of susceptible population with a constant (imposed) and infection oriented interventions. Epidemiological and demographic data are used to estimate the model parameters. The baseline case was explored further to showcase several critical and predictive scenarios.

Results and analysis: The model simulations are in good agreement with the infection data for the period of 5 March 2020 to 31 January 2021. Dynamic isolation and screening program are found to be potential measures that can alter the course of epidemic. A $7 \%$ increase in isolation rate may result in a $31 \%$ reduction of epidemic peak whereas a 3 times increase in screening rate may reduce the epidemic peak by $35 \%$. The model predicts that nearly $9.7 \%$ to $12 \%$ of the total population of New Jersey may become infected within the middle of July 2021 along with 24.6 to 27.3 thousand cumulative deaths. Within a wide spectrum of probable scenarios, there is a possibility of third wave. Conclusion: Our findings could be informative to the public health community to contain the pandemic in the case of economy reopening under a limited or no vaccine coverage. Additional epidemic waves can be avoided by appropriate screening and isolation plans.

\section{INTRODUCTION}

Public health communities are facing unprecedented challenges to control the SARS-CoV-2 pandemic, largely, due to the limitation of effective vaccines and lack of therapeutic treatment. In the absence of vaccines, nonpharmaceutical interventions such as screening, isolation, social distancing, quarantine, maintaining public hygiene (wearing mask and washing hands frequently) and contact tracing are in place to curtail the pandemic [7. Although these measurements are highly potential to eliminate a pandemic, they are not socioeconomically friendly and may become impracticable for a long run. A lack of proper implementation of these interventions may turn the effort into a futile exercise as it has been seen from the current pandemic scenarios of SARS-CoV-2 in different countries [14].

Mathematical models have been used to assess the effectiveness of the control measures and to guide the public health policy. Models help to understand the dynamics of infectious diseases under the given circumstances and provide valuable information about how to control the disease. The impact of nonpharmaceutical interventions against the spread of SARS-CoV-2 can be seen in numerous studies [19, 23, 26]. Some studies showed that mitigation, plans and preparedness should be organized and

Received by the editors 23 April 2021; accepted 2 October 2021; published online 10 October 2021.

2010 Mathematics Subject Classification. Primary 92D30, 37M05; Secondary 34A34, 34H05.

Key words and phrases. SARS-CoV-2, screening, dynamic isolation, basic reproduction number, economy reopening, final size. 
deployed globally to prevent the progression of transmission rate, otherwise the basic reproduction number could reach up higher [16, 34, 43, 44]. A study on the basis of diagnosis and treatment resources indicated that a delayed diagnosis is somehow responsible for the increased transmission risk [31] whereas improvement of detection rate can rapidly and significantly lessen the death rate [40. An investigation suggests that besides the medical facilities, the effectiveness of early media coverage may increase the public awareness [45]. A research focusing on the influenza type patients considered the seasonal pattern of SARS-CoV-2 into account and revealed that enhancement of mass influenza vaccination and public health interventions have positive impacts to contain the outbreak 20].

Studies found that implementation of quarantine strategies [27] and other important measures such as setting up complete lockdown with isolation, confirming media coverage and ensuring public hygiene can significantly mitigate the severity of the outbreak 22, more specifically, implementation of lockdown and centralized quarantine policy play a significant role in reducing the infection [35. Recent models also pointed out some of the important control policies, for example, the effect of emergency and healthy sanitary measures [1, the impact of social distancing [10, 13, the outcome of estimating intensive care unit [25] and providing adequate medical resources like protective clothing, health workforce and necessary medicines [39 in order to reduce the community transmissions of SARS-CoV-2 infection. Besides nonpharmaceutical interventions, vaccination process is in progress and underway [41. The current vaccination rates can minimize the infection but control policies also need to go on [30. Moreover, strategies for optimum vaccine allocation depend on numerous factors [11.

In order to complement other studies, we would like to see the effect of population behaviour on the pandemic. An important aspect of the pandemic is the behaviour of the population due to the fear of severe consequences such as death or long term sickness. In general, the behaviour changes with the reported cases and incidence. People may go to self isolation and return from there subject to the intensity of these numbers. In contrast, an economy reopening program may increase the new infections through the elevated contacts. To contain the infection in a circumstance of the elevated contacts, the screening program may help to reduce infections (as it detects the asymptomatic cases and puts them in quarantine [12]) and can significantly mitigate the outbreak besides other protective measures [4. We aim to identify the interplay of dynamic isolation, screening program and infection through a mathematical model.

To properly explore the effects of screening program and dynamic isolation, we develop a SARS-CoV2 model that takes the Kermack-McKendrick form [15] with dynamic population movement. We use a SARS-CoV-2 data set of New Jersey, USA (cumulative reported deaths and cumulative reported cases) 42. to parameterize and validate the model. The remaining part of the paper is organized as follows: a mathematical model is developed in Section 2 Section 3 is dedicated to data collection, parameter estimation and data fitting. Section 4 presents the results and model simulations. Finally, Section 5 discusses the findings, consequences and uncertainties.

\section{MODEL}

This section is dedicated to develop a mathematical model for capturing the dynamics of SARSCoV-2 infection. We also present an elementary analysis of the model and derive useful thresholds.

2.1. Model formulation. We consider $N(t)$ as the total population and divide it into seven different classes (compartments): $S(t)$, susceptible; $A(t)$, asymptomatic (undetected); $A_{q}(t)$, quarantined asymptomatic (detected through screening); $S_{o}(t)$, isolated; $I(t)$, infected (symptomatic but undetected); $I_{q}(t)$, quarantined (symptomatic and detected); and $R(t)$, recovered. Infection is spread by the individuals from $A, A_{q}, I$ and $I_{q}$ classes with different transmission rates. The susceptible population become infected due to effective contacts with the asymptomatic (both undetected and detected) and 
the symptomatic individuals (infected and quarantined) who carry the pathogen. As a result, susceptible individuals $(S)$, at first, move to the asymptomatic stage $(A)$ with an effective transmission rate $(\beta)$. The individuals in the asymptomatic stage $(A)$ are clinically silent and thus remain undetected unless tested through a screening program. Individuals from the undetected asymptomatic stage may develop symptoms and become fully infectious (undetected) in course of time, thus move to the infected stage $(I)$ at the rate of $\eta_{1}$. A portion of the asymptomatic individuals undergo diagnosis through screening program and become quarantined asymptomatic $\left(A_{q}\right)$ with a rate $\sigma$. Individuals move from $A_{q}$ to $I_{q}$ by disease progression at a rate $\eta_{2}$ and from $I$ to $I_{q}$ through diagnosis at a rate $\tau$. Due to immunity or treatment, individuals who are in stages $A, A_{q}, I$ and $I_{q}$ may get recovery from the infection with the recovery rates $\gamma_{1}, \gamma_{2}, \gamma_{3}$ and $\gamma_{4}$, respectively. We assume that a portion of total population remains isolated $\left(S_{o}\right)$ due to awareness or fear. The isolation continues with variation throughout the observation period with a dynamic rate $\alpha_{+}$subject to the number of reported cases. In other words, individuals from susceptible class may go for isolation at any time due to fear or due to the regulations (for example, a lockdown).

The isolation rate $\left(\alpha_{+}\right)$and the transmission rate $(\beta)$ are considered to be nonlinear with respect to the reported cases. The isolation rate is an increasing function of the reported cases and in contrast, the effective transmission rate is a decreasing function of the reported cases. In particular, we consider

$$
\alpha_{+}=a_{+} \exp \left(\psi_{1} \zeta\right), \quad \beta=\beta_{o} \exp \left(-\psi_{2} \zeta\right)
$$

where $\zeta$ is the daily reported cases, $a_{+}$and $\beta_{o}$ are the initial values of isolation rate and effective transmission rate and $\psi_{i}: i=1,2$ are constants.

We assume that isolated individuals are unlikely to get infection unless they move back to $S$ class with a rate of $\alpha_{-}$. Our assumptions also include that (i) there is no immigration (population size does not change significantly over the observation period), (ii) vaccination is ignored (since vaccination campaign is still in progress and underway, (iii) recovery gives a permanent immunity, which means, the possibility of becoming second time susceptible after getting recovery from the disease is omitted based on prior evidence [5, 18, (iv) death occurs only in $I$ and $I_{q}$ compartments due to the disease with rates $\delta_{1}$ and $\delta_{2}$, respectively; other deaths are ignored and (v) quarantined individuals have very little contribution to the reported cases (that is, $\epsilon_{1}, \epsilon_{2}<<\epsilon_{3}$ ).

The population movement discussed above is presented in Figure 1 and the descriptions of variables and parameters are summarized in Tables 1 and 2 respectively. Considering the above assumptions, the transmission dynamics of SARS-CoV-2 can be modeled by the following system of ordinary differential equations:

$$
\left\{\begin{aligned}
\dot{S} & =-\beta\left(A+\epsilon_{1} A_{q}+\epsilon_{3} I+\epsilon_{2} I_{q}\right) S-\alpha_{+} S+\alpha_{-} S_{o} \\
\dot{A} & =\beta\left(A+\epsilon_{1} A_{q}+\epsilon_{3} I+\epsilon_{2} I_{q}\right) S-\left(\eta_{1}+\sigma+\gamma_{1}\right) A \\
\dot{A_{q}} & =\sigma A-\left(\gamma_{2}+\eta_{2}\right) A_{q} \\
\dot{S}_{o} & =\alpha_{+} S-\alpha_{-} S_{o} \\
\dot{I} & =\eta_{1} A-\left(\delta_{1}+\tau+\gamma_{3}\right) I \\
\dot{I}_{q} & =\tau I+\eta_{2} A_{q}-\left(\delta_{2}+\gamma_{4}\right) I_{q} \\
\dot{R} & =\gamma_{1} A+\gamma_{2} A_{q}+\gamma_{3} I+\gamma_{4} I_{q}
\end{aligned}\right.
$$

where $N=S+A+A_{q}+S_{o}+I+I_{q}+R$.

To track the SARS-CoV-2 related deaths and cases (requisite for adjusting model 2.1) with realistic data for predicting and measuring the public health impact of the disease), we define two state variables: $C_{r d}$, cumulative reported deaths; and $C_{r c}$, cumulative reported cases. We assume that the data that 


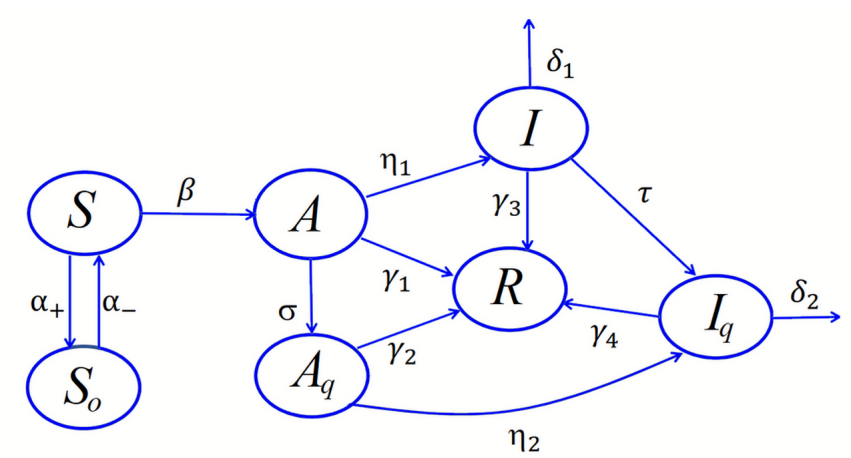

Figure 1. Transmission diagram of model 2.1) representing the movement of population based on the disease status.

TABLE 1. Variables used in model (2.1).

\begin{tabular}{ll}
\hline Variable & Description \\
\hline$S$ & Number of susceptible individuals \\
$A$ & Number of asymptomatic individuals \\
$A_{q}$ & Number of quarantined asymptomatic individuals \\
$S_{o}$ & Number of isolated individuals \\
$I$ & Number of infected individuals \\
$I_{q}$ & Number of quarantined individuals \\
$R$ & Number of recovered individuals \\
$C_{r d}$ & Cumulative reported deaths \\
$C_{r c}$ & Cumulative reported cases \\
$N$ & Total population \\
\hline
\end{tabular}

TABle 2. Parameters used in model (2.1).

\begin{tabular}{ll}
\hline Parameter & Description \\
\hline$\beta_{o}$ & Initial value of effective transmission rate \\
$\epsilon_{1}$ & Transmission rate for $A_{q}$ \\
$\epsilon_{2}$ & Transmission rate for $I_{q}$ \\
$\epsilon_{3}$ & Transmission rate for $I$ \\
$a_{+}$ & Initial value of isolation rate \\
$\alpha-$ & Rate at which isolated individuals move back to $S$ \\
$\sigma$ & Screening program rate \\
$\eta_{1}$ & Incubation rate \\
$\eta_{2}$ & Rate at which quarantined asymptomatic individuals move to $I_{q}$ \\
$\tau$ & Transition rate from $I$ to $I_{q}$ \\
$\gamma_{1}$ & Recovery rate of $A$ \\
$\gamma_{2}$ & Recovery rate of $A_{q}$ \\
$\gamma_{3}$ & Recovery rate of $I$ \\
$\gamma_{4}$ & Recovery rate of $I_{q}$ \\
$\delta_{1}$ & Disease induced death rate in $I$ \\
$\delta_{2}$ & Disease induced death rate in $I_{q}$ \\
$\psi_{i}$ & Adjustment coefficients $(i=1,2)$ \\
\hline
\end{tabular}

are publicly available are based on the detected cases [42. Our model 2.1] comprises with two detected compartments: $A_{q}$ and $I_{q}$. Thus, the dynamics of $C_{r d}$ and $C_{r c}$ can be obtained from model (2.1) as follows:

$$
\begin{aligned}
& \dot{C_{r d}}=\delta_{2} I_{q}, \\
& \dot{C_{r c}}=\sigma A+\tau I .
\end{aligned}
$$


2.2. Well-posedness. The model 22.1) is a system of seven coupled equations. Following [28, 29, it can be shown that the state variables presented in the model yield non-negative values for $t \geq 0$ provided that the initial values stand non-negative. It also follows from model (2.1) that $\dot{S}(t)+\dot{A}(t)+$ $\dot{A}_{q}(t)+\dot{S}_{o}(t)+\dot{I}(t)+\dot{I}_{q}(t)+\dot{R}(t)=-\delta_{1} I-\delta_{2} I_{q}$. That is, in the absence of infection, $\dot{N}=0$ (which means, $N(t)$ is constant).

2.3. Basic reproduction number. The basic reproduction number is a threshold that determines whether an epidemic is in increasing or decreasing trends. It is usually denoted by the symbol $\Re_{o}$. If $\Re_{o}$ is larger than unity, then the number of reported cases remain in upward direction. For our model (2.1), there exists a disease free equilibrium point denoted by $\xi_{o}=\left(\bar{S}, 0,0, \bar{S}_{o}, 0,0,0\right)$ where $\bar{S}=\frac{\alpha_{-} S_{o}}{a_{+}}$. Using the next generation matrix approach [37, we can obtain the basic reproduction number $\left(\Re_{o}\right)$. It involves two major components, the new infection matrix $(F)$ and the transfer matrix $(V)$ which, for our model (2.1) at $\xi_{o}$, are given by

$$
\begin{aligned}
F & =\left(\begin{array}{cccc}
\beta_{o} \bar{S} & \beta_{o} \epsilon_{1} \bar{S} & \beta_{o} \epsilon_{3} \bar{S} & \beta_{o} \epsilon_{2} \bar{S} \\
0 & 0 & 0 & 0 \\
0 & 0 & 0 & 0 \\
0 & 0 & 0 & 0
\end{array}\right) \\
V & =\left(\begin{array}{cccc}
\eta_{1}+\sigma+\gamma_{1} & 0 & 0 & 0 \\
-\sigma & \gamma_{2}+\eta_{2} & 0 & 0 \\
-\eta_{1} & 0 & \delta_{1}+\tau+\gamma_{3} & 0 \\
0 & -\eta_{2} & -\tau & \delta_{2}+\gamma_{4}
\end{array}\right)
\end{aligned}
$$

Then $\Re_{o}$ can be written as follows:

or

$$
\Re_{o}=\frac{\bar{S} \beta_{o}}{c_{1}}+\frac{\bar{S} \beta_{o} \sigma \epsilon_{1}}{c_{1} c_{3}}+\frac{\bar{S} \beta_{o} \eta_{1} \epsilon_{3}}{c_{1} c_{2}}+\frac{\bar{S} \beta_{o} \epsilon_{2}\left(\tau \eta_{1} c_{3}+\sigma \eta_{2} c_{2}\right)}{c_{1} c_{2} c_{3} c_{4}}
$$

$$
\Re_{o}=\left(\frac{\beta_{o}}{c_{1}}+\frac{\beta_{o} \sigma \epsilon_{1}}{c_{1} c_{3}}+\frac{\beta_{o} \eta_{1} \epsilon_{3}}{c_{1} c_{2}}+\frac{\beta_{o} \epsilon_{2}\left(\tau \eta_{1} c_{3}+\sigma \eta_{2} c_{2}\right)}{c_{1} c_{2} c_{3} c_{4}}\right) \frac{\alpha_{-} \bar{S}_{o}}{a_{+}}
$$

where

$$
c_{1}=\sigma+\gamma_{1}+\eta_{1}, c_{2}=\tau+\gamma_{3}+\delta_{1}, c_{3}=\gamma_{2}+\eta_{2} \text { and } c_{4}=\gamma_{4}+\delta_{2} .
$$

Specifically from (2.4), we have the following:

$$
\Re_{o}=\Re_{o}^{A}+\Re_{o}^{A_{q}}+\Re_{o}^{I}+\Re_{o}^{I_{q}}
$$

The four terms on the right hand side of 2.5 demonstrate the transmission routes from asymptomatic $(A)$ to susceptible $(S)$, quarantined asymptomatic $\left(A_{q}\right)$ to susceptible $(S)$, infected $(I)$ to susceptible $(S)$ and quarantined $\left(I_{q}\right)$ to susceptible $(S)$, respectively. All these four modes of transmission contribute to $\Re_{o}$ and are collectively responsible to shape the total infection risk of SARS-CoV-2.

2.4. Final size. The final size $(\mathcal{F})$ is a critical indicator for a pandemic. It shows the overall impact of the pandemic on the population. Thus, one would be interested to reduce the final size by controlling the model parameters. For a nonlinear model, the final size may not be expressed explicitly. In order to derive the final size of the pandemic, first we need to have the final size equation by introducing the number of susceptible individuals right at the end of the outbreak. We follow the method discussed in [3] to find the final size equation. We consider that $S_{\infty}$ shapes the end of the outbreak which is the limit of $S(t)$ as $t \rightarrow \infty$. For such, let $x \in \mathbb{R}^{n}$ be the set of infected components, $y \in \mathbb{R}^{m}$ the set of susceptible component and $z \in \mathbb{R}^{k}$ the set of isolated and recovered components. Thus from model 2.1., we have $x(t)=\left(A(t), A_{q}(t), I(t), I_{q}(t)\right)^{T}, y(t)=S(t)$ and $z(t)=\left(S_{o}(t), R(t)\right)^{T}$. Let $b$ be 
the row vector representing the horizontal transmission. Therefore, we have $m=1, n=4, k=2$ and $b=\left(1, \epsilon_{1}, \epsilon_{3}, \epsilon_{2}\right)$. Then, we obtain the following result:

Theorem 2.1. The final size equation of model 2.1) at $\xi_{o}$ is given by

$$
\ln \left(\frac{y_{o}}{y_{\infty}}\right)=\Re_{o} \frac{y_{o}-y_{\infty}}{y_{o}}+\beta_{o} b V^{-1} x_{o}
$$

Equivalently, we have

$$
\ln \left(\frac{\bar{S}}{S_{\infty}}\right)=\Re_{o} \frac{\bar{S}-S_{\infty}}{\bar{S}}+\beta_{o}\left(A(0) g_{1}+A_{q}(0) g_{2}+I(0) g_{3}+I_{q}(0) g_{4}\right)
$$

where

$$
g_{1}=\frac{1}{c_{1}}+\frac{\sigma \epsilon_{1}}{c_{1} c_{3}}+\frac{\epsilon_{3} \eta_{1}}{c_{1} c_{2}}+\frac{\epsilon_{2}\left(\tau c_{3} \eta_{1}+\sigma c_{2} \eta_{2}\right)}{c_{1} c_{2} c_{3} c_{4}}, g_{2}=\frac{\epsilon_{1}}{c_{3}}+\frac{\epsilon_{2} \eta_{2}}{c_{3} c_{4}}, g_{3}=\frac{\tau \epsilon_{2}}{c_{2} c_{4}}+\frac{\epsilon_{3}}{c_{2}} \text { and } g_{4}=\frac{\epsilon_{2}}{c_{4}} .
$$

Now following [24], the final size $(\mathcal{F})$ of the pandemic (which is equal to the final size of the susceptible population $\left(\mathcal{F}_{\mathcal{S}}\right)$ ) is the total number of individuals (either dead or recovered) who have experienced the SARS-CoV-2 infection. Therefore, we have

$$
\mathcal{F}=\mathcal{F}_{\mathcal{S}}
$$

where

$$
\mathcal{F}_{\mathcal{S}}=\bar{S}-S_{\infty} \text { or } \mathcal{F}_{\mathcal{S}}=\frac{\alpha_{-} \bar{S}_{o}}{a_{+}}-S_{\infty}
$$

Equation 2.8 can be used to control the final size of the pandemic by manipulating the control parameters. Details will be explored in Section 4.

\section{Data And parameter estimation}

3.1. Data. SARS-CoV-2 data set of New Jersey [42] is used to validate and parameterize the model 2.1. In particular, we extracted cumulative reported deaths and cumulative reported cases from the data set occurred in New Jersey from 5 March 2020 to 31 January 2021.

3.2. Initial conditions and parameter values. We consider the total population of New Jersey [36] as the initial population size, i.e., $N(0)=8882190$. We set $S=6749674$ and accordingly $S_{o}=2131726$. From data fitting, we found $A(0)=788$. Considering the reported cases of 5 March 2020, $I(0)=2$ and $R(0)=0$. At the beginning of the observation period, we consider that no infected individuals were in quarantine and therefore $A_{q}(0)=0$ and $I_{q}(0)=0$. The range of incubation period varies from 2 to 14 days [44]. Considering the mean incubation period of 7 days, we obtain $\eta_{1}=1 / 7$. Study shows that within 7 days (a median time) from the first symptom, patients move to hospital admission or quarantine [38, which yields $\eta_{2}=1 / 7$. On the other hand, it may take about two weeks to recover from the infections [31, 32, 44] and thus we obtain $\gamma_{1}=\gamma_{2}=\gamma_{3}=\gamma_{4}=1 / 14$. It is also reported that the waiting time of the infected individuals for timely diagnosis ranges between 1 to 5 days. That means, the infected individuals move to quarantine within 1 to 5 days [31] and so we take 3 days for the transition period from $I$ to $I_{q}$ class, i.e., $\tau=1 / 3$. The set of different parameter values is listed in Table 3 . 
TABLE 3. Values of the parameters.

\begin{tabular}{llll}
\hline Parameter & Baseline Value & Unit & Source \\
\hline$\beta_{o}$ & $3.4 \times 10^{-8}$ & per day & Fitted \\
$\epsilon_{1}$ & 0.65 & per day & Fitted \\
$\epsilon_{2}$ & 0.43 & per day & Fitted \\
$\epsilon_{3}$ & 1.15 & per day & Fitted \\
$a_{+}$ & 0.0011 & per day & Fitted \\
$\alpha_{-}$ & 0.0034 & per day & Fitted \\
$\sigma$ & 0.011 & per day & Fitted \\
$\eta_{1}$ & $1 / 7$ & per day & 44 \\
$\eta_{2}$ & $1 / 7$ & per day & 38 \\
$\tau$ & $1 / 3$ & per day & 31 \\
$\gamma_{i} ; i=1,2,3,4$ & $1 / 14$ & per day & 31 \\
$\delta_{1}$ & 0.01 & per day & Fitted \\
$\delta_{2}$ & 0.0075 & per day & Fitted \\
$\psi_{1}$ & $9.5 \times 10^{-4}$ & dimensionless & Fitted \\
$\psi_{2}$ & $3.5 \times 10^{-5}$ & dimensionless & Fitted \\
\hline
\end{tabular}

3.3. Data fitting. The model (2.1) is fitted to the pandemic data set [42] so that the parameters $\beta_{o}, \epsilon_{1}, \epsilon_{2}, \epsilon_{3}, \sigma, a_{+}, \alpha_{-}, \psi_{1}, \psi_{2}, \delta_{1}, \delta_{2}$ and the initial value $A(0)$ can be estimated. Since the asymptomatic cases were unknown, we found it reasonable to estimate its initial value. To estimate the unknowns, first, the model (2.1) is solved by using the MATLAB built-in function ode45 with some initial guesses. Then, the solutions are compared to the given data by fmincon (MATLAB routine) to estimate the parameters. More precisely, we minimize the following error function:

$$
E=\sum_{i=1}^{333}\left[\left(C_{r d}\left(t_{i}\right)-\hat{C_{r d}}\left(t_{i}\right)\right)^{2}+\left(C_{r c}\left(t_{i}\right)-\hat{C_{r c}}\left(t_{i}\right)\right)^{2}\right]
$$

where $C_{r d}\left(t_{i}\right)$ and $C_{r c}\left(t_{i}\right)$ are the solutions of $(2.2)$ computed numerically at time $t_{i}$ and $\hat{C_{r d}}\left(t_{i}\right)$ and $\hat{C_{r c}}\left(t_{i}\right)$ are the corresponding data. Here, $i=1$ and $i=333$ represent 5 March 2020 and 31 January 2021, respectively.

Due to the dynamic isolation rate and effective transmission rate described in Section 2 model 2.1) fits very well to the large data set (cumulative reported deaths and cumulative reported cases). The nonlinear functions used for the dynamic isolation rate and effective transmission rate are: $\alpha_{+}=$ $a_{+} e^{\psi_{1}[\sigma A+\tau I]}$ and $\beta=\beta_{o} e^{-\psi_{2}[\sigma A+\tau I]}$, respectively. Figure 2 shows the fitting result of the model (2.1) to the cumulative reported deaths and cumulative reported cases occurred in New Jersey from 5 March 2020 to 31 January 2021. It is observed that the predictions given by the model (2.1) represent a trend which is very similar to the reported cases [42]. Therefore, dynamic isolation rate and effective transmission rate seem to be reasonable and realistic factors that need to be explored. Two pandemic waves of infection are seen from data fitting, one of which (first wave) occurred in April 2020 and another one (second wave) occurred in January 2021. Owing to the first and second waves of infection, nearly 0.7 million cumulative reported cases (which is $7.8 \%$ of the total population of New Jersey) and 22 thousand cumulative reported deaths are estimated on 31 January 2021. The results obtained through this data fitting are considered as the base case. If infection trend follows the same pattern after 31 January 2021 as it is in the base case, our model, in consequence, projects nearly 2.6 thousand additional deaths (24.6 thousand deaths in total) and 0.16 million additional cases ( 0.86 million cases in total which is $9.7 \%$ of the total population of New Jersey) within 18 July 2021. Such projection is considered as the current projection. 

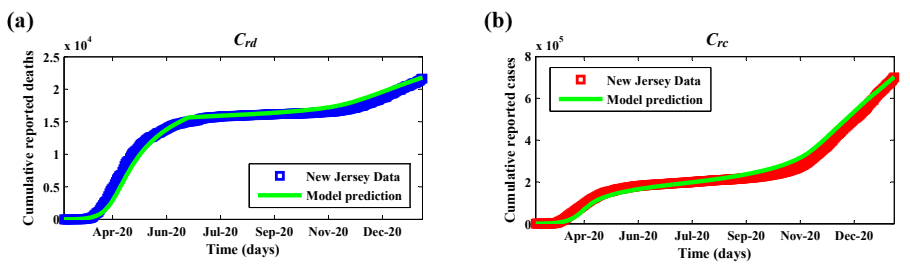

Figure 2. Model fitting to New Jersey data from 5 March 2020 to 31 January 2021. (a) The squares (blue) display the cumulative reported deaths and the solid curve (green) represents the model prediction. (b) The squares (red) display the cumulative reported cases and the solid curve (green) represents the model prediction.

\section{REsults}

4.1. Sensitivity of $\Re_{o}$ on model parameters. Based on data fitting and estimated parameters, the basic reproduction number of our model $(2.1)$ is: $\Re_{o}=2.06$. Among the four infectious components ( $\left.A, A_{q}, I, I_{q}\right)$, the largest magnitude of $\Re_{o}$ is observed in asymptomatic $(A)$ to susceptible $(S)$ transmission (i.e., $\Re_{o}^{A}$ ). Since asymptomatic individuals are clinically silent, they transmit the infection to others by coming in close contact unknowingly. The smallest magnitude of $\Re_{o}$ arises from quarantined asymptomatic $\left(A_{q}\right)$ to susceptible $(S)$ transmission (i.e., $\left.\Re_{o}^{A_{q}}\right)$. This is because of the screening program which detects the asymptomatic cases and immediately sends them to quarantine. The basic reproduction number includes several key parameters. The sensitivity analysis of $\Re_{o}$ could inform how significant each parameter is to the disease transmission [8, 25. This analysis is generally useful to understand the robustness of model projections to the parameter values and to discover the specific parameters of a model which have high impacts on $\Re_{o}$. Such specific parameters can be targeted for control or intervention policies. To identify the critical parameters, first, we define the sensitivity index.

Definition 4.1. The normalized forward sensitivity index of $\Re_{o}$ is differentiable with respect to a specified parameter $p$ and is defined by

$$
\Upsilon_{p}^{\Re_{o}}=\frac{\partial \Re_{o}}{\partial p} \frac{p}{\Re_{o}}
$$

The parameter values from Table 3 are used to determine the sensitivity indices which are listed in Table 4. The positive indices in Table 4 indicate that the corresponding parameters will cause to increase $\Re_{o}$. On the contrary, the parameters with negative sensitivity indices will reduce $\Re_{o}$. This is explored in Figure 3 which demonstrates the significance of some important parameters to $\Re_{o}$. It is understood that $\Re_{o}$ can significantly be reduced through the implementation of isolation, acceleration of screening program and by minimizing the transmission rate. Moreover, the combined effects of isolation and screening program on $\Re_{o}$ is also noteworthy.

4.2. Computation of the final size. Equation 2.7) is used to see the effects of isolation and screening program on $S_{\infty}$ (see Figure 4). It is worth mentioning that $S_{\infty}$ will be larger if the values of isolation and screening parameters are increased and thus final size can eventually be reduced. The numerical value of $S_{\infty}$ for our model 2.1 ) is found to be $1.19 \times 10^{6}$ (which is the approximate number of susceptible individuals right at the end of the pandemic) for the baseline values of screening and isolation parameters. Using equation 2.8 , the final size $(\mathcal{F})$ of the pandemic is calculated to be $5.4 \times 10^{6}$, which means, nearly $60 \%$ of the total population is likely to be infected (both reported and unreported) by the end of the pandemic. 
TABLE 4. Sensitivity of $\Re_{o}$.

\begin{tabular}{ll}
\hline Parameter & Sensitivity index \\
\hline$\beta_{o}$ & +1.0000 \\
$\epsilon_{1}$ & +0.0159 \\
$\epsilon_{2}$ & +0.3176 \\
$\epsilon_{3}$ & +0.1891 \\
$\alpha_{-}$ & +1.0000 \\
$a_{+}$ & -1.0000 \\
$\sigma$ & -0.0224 \\
$\eta_{1}$ & -0.1465 \\
$\eta_{2}$ & -0.0043 \\
$\tau$ & -0.0940 \\
$\gamma_{1}$ & -0.3171 \\
$\gamma_{2}$ & -0.0117 \\
$\gamma_{3}$ & -0.0840 \\
$\gamma_{4}$ & -0.2875 \\
$\delta_{1}$ & -0.0118 \\
$\delta_{2}$ & -0.0302 \\
$\psi_{1}$ & -0.0089 \\
$\psi_{2}$ & -0.0003 \\
\hline
\end{tabular}

(a)

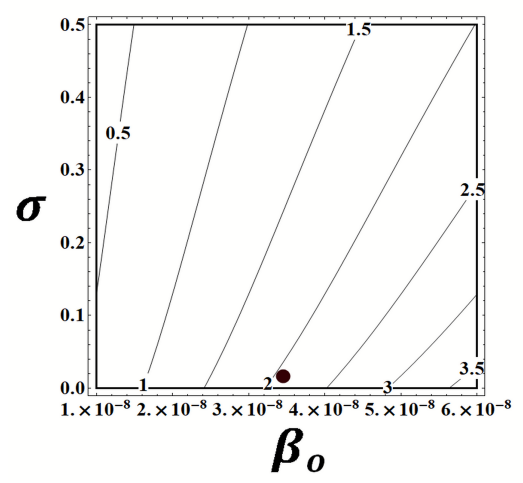

(c)

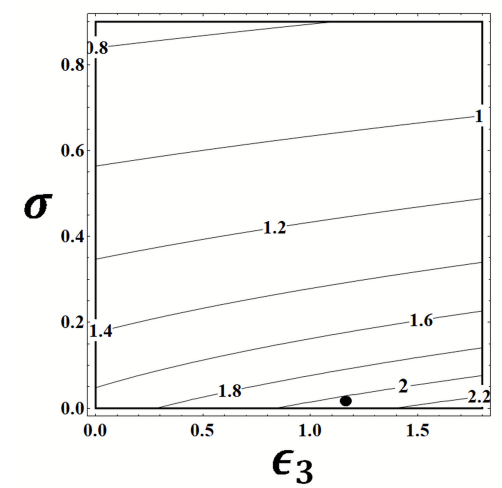

(b)

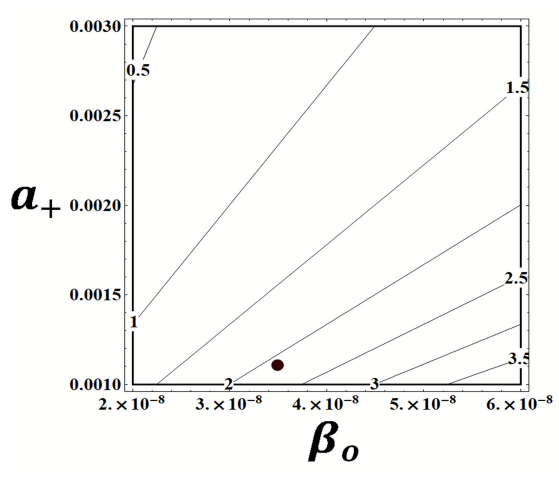

(d)

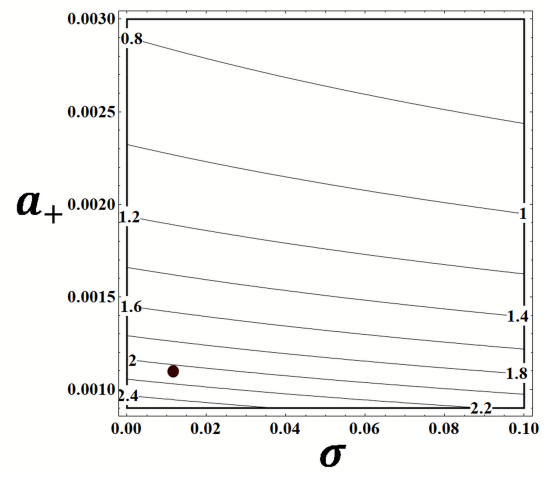

FiguRE 3. Contour plots of basic reproduction number $\left(\Re_{o}\right)$ by (a) varying $\beta_{o}$ and $\sigma$, (b) varying $\beta_{o}$ and $a_{+},(\mathrm{c})$ varying $\epsilon_{3}$ and $\sigma,(\mathrm{d})$ varying $\sigma$ and $a_{+}$. Base case is pointed with closed circles (black). The remaining parameter values are kept fixed, given in Table 3 


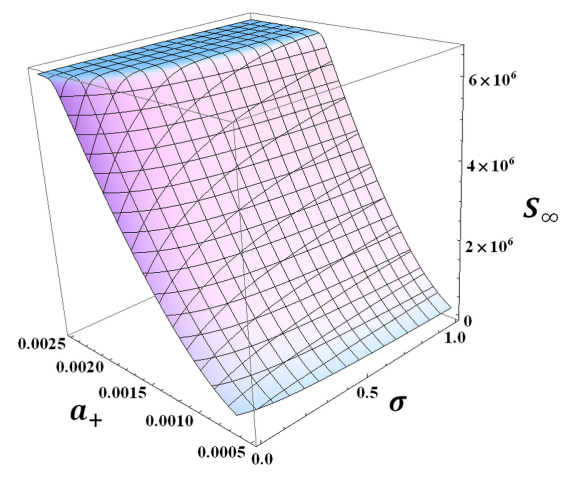

Figure 4. Susceptible individuals right at the end of the pandemic $\left(S_{\infty}\right)$ varying $\sigma$ and $a_{+}$. The remaining parameter values are kept fixed, given in Table 3.

4.3. Effects of screening program. Screening is effective for detection of the asymptomatic cases. According to our model, a 3 times increase of screening compared to the base case from 5 March 2020 to 31 January 2021 could reduce the first wave of infection by $35 \%$ and second wave of infection by $25 \%$ relative to the base case (not shown in a Figure). Our model further predicts the possible impact of screening program on the epidemic from 31 January 2021 to 18 July 2021 (see Figure 5). It shows that a $73 \%$ increase of the screening program may reduce the cumulative reported deaths and cumulative reported cases by $4 \%$ and $9 \%$, respectively for this period. The model also predicts that a $90 \%$ increase of screening program (not shown in a Figure) will possibly eliminate the infection fully and therefore cumulative reported deaths and cumulative reported cases can be minimized by $5 \%$ and $12 \%$, respectively.

4.4. Effects of dynamic isolation. Isolation is a key tool to curve the epidemic. Our model estimates the effects of isolation with different schemes. A $7 \%$ increase of isolation compared to the base case (from 5 March 2020 to 31 January 2021) could reduce the first wave of infection by $31 \%$ and the second wave by $23 \%$ relative to the base case (not shown in a Figure). Figure 6 shows a scenario to demonstrate the effects of an increased isolation from 31 January 2021 to 18 July 2021 . It reveals that a $16 \%$ increase of isolation from the base case may cause to eliminate the infection by 18 July 2021 (assuming the other interventions at the base line). Such additional isolation can reduce $4 \%$ of cumulative reported deaths and $10 \%$ of cumulative reported cases by 18 July 2021 compared to the base case scenario.

4.5. Combined effects of isolation and screening program. Figure 3 (d) demonstrates the mutual effects of isolation and screening program on $\Re_{o}$. It shows that both isolation and screening rates should be increased in order to stop the spread of the infection. For example, if isolation is increased by $80 \%$ and screening by 9 times from the base case, then $\Re_{o}$ will be less than 1 . Likewise, isolation and screening can be increased by $90 \%$ and 4 times, respectively from the base case to keep $\Re_{o}$ below 1 . On the other hand, if isolation decreases, then screening rate needs to be increased so that $\Re_{o}$ can be made below 1 . For example, if isolation rate is decreased by $5 \%$, screening rate, at that time, should be increased by $50 \%$ to maintain the $\Re_{o}$ value. Similarly, while isolation drops about $10 \%$ from the base case, screening rate should be greater than $60 \%$ for $\Re_{o}$ to be less than 1 .

4.6. Possibility of third wave. While the current epidemic appears to be an end by July 2021, there is a possibility of resurgence of epidemic if the undertaken measurements are loosened significantly. Figure 7 shows that a decline of $15 \%$ isolation rate after 31 January 2021 may trigger the infection 
(a)

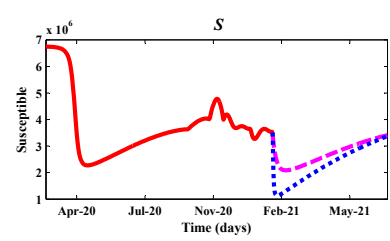

(d)

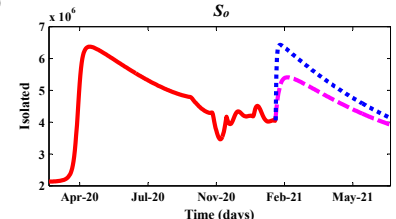

(g)

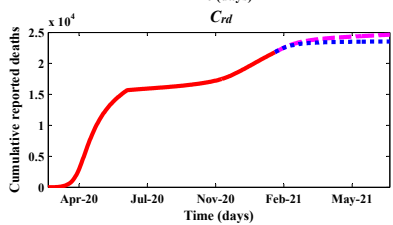

(b)

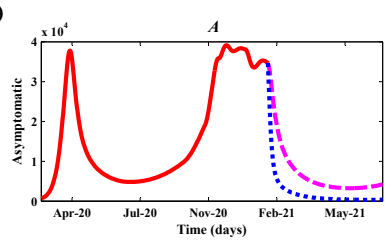

(e)

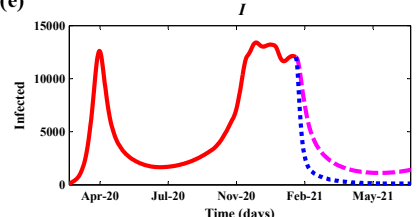

(h)

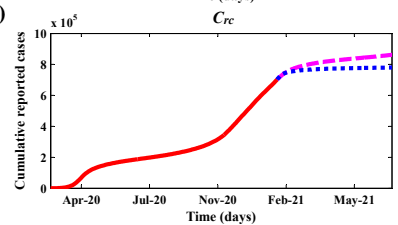

(c)

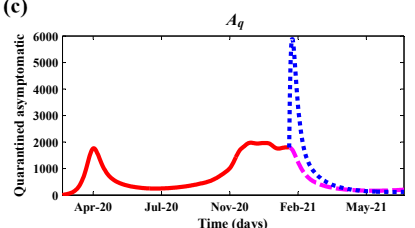

(f)
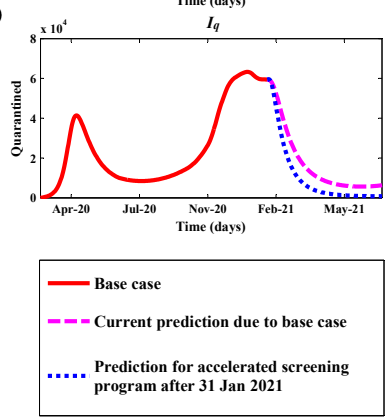

FiguRE 5. Projections for screening program. Model simulations from 5 March 2020 to 18 July 2021 for (a) susceptible class, (b) asymptomatic class, (c) quarantined asymptomatic class, (d) isolated class, (e) infected class, (f) quarantined class, (g) cumulative reported deaths and (h) cumulative reported cases. The solid curves (red) represent the base case whereas the dashed curves (magenta) represent the current projections following the pattern of base case and the dotted curves (blue) represent the projections upto 18 July 2021 due to $73 \%$ increase of screening from the base case. The parameter values are given in Table 3

which can cause the third wave of SARS-CoV-2 pandemic in New Jersey with a possible pandemic peak in between 30 March 2021 to 11 April 2021. As a result, 2.7 thousand additional deaths and 0.21 million additional cases may occur by 18 July 2021 (compared to the current projection of 18 July 2021). A lack of screening program may also cause the resurgence of epidemic as shown in Figure 8. It shows that a $80 \%$ reduction of screening program (after 31 January 2021 till 18 July 2021) may cause to increase the infection rapidly in New Jersey with a possible pandemic peak in the middle of April 2021. According to our model simulations, nearly 2.68 thousand additional deaths and 0.18 million additional cases may occur within 18 July 2021 (compared to the current projection of 18 July 2021) for such reduction of screening program.

\section{Discussion}

This study evaluates some nonpharmaceutical interventions against the spread of SARS-CoV-2 through a mathematical model. The model is unique in a sense that it incorporates a natural phenomenon of population movement during a pandemic. It also shows an outstanding consistency with SARS-CoV-2 data set for a period of over ten months.

The results show that an accelerated screening program can reduce the infection significantly which is reasonable as the detected cases are taken to quarantine. However, it is important to quantify the required effort which is shown by our model that more than $70 \%$ acceleration of screening program may reduce the cumulative reported deaths and cumulative reported cases by $4 \%$ and $9 \%$, respectively. Moreover, a $85 \%$ to $90 \%$ acceleration of screening program from the base case can fully eliminate the disease. These findings are consistent with a prior work [2]. 
(a)

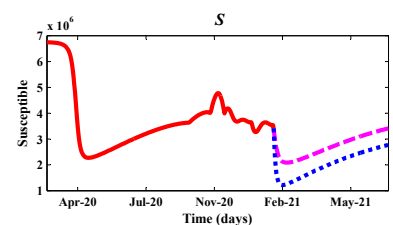

(d)

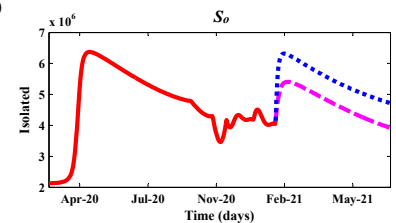

(g)

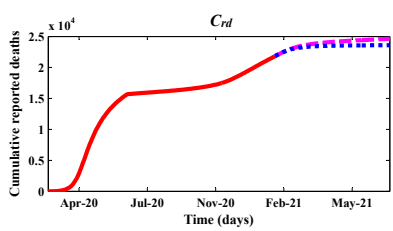

(b)

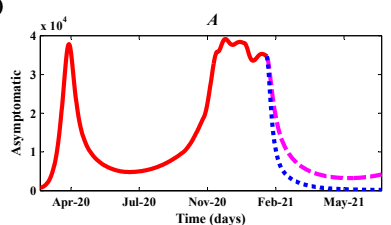

(e)

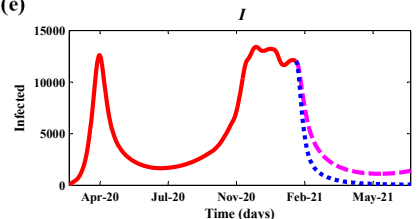

(h)

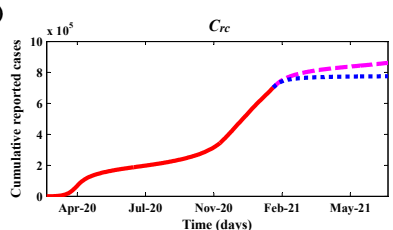

(c)

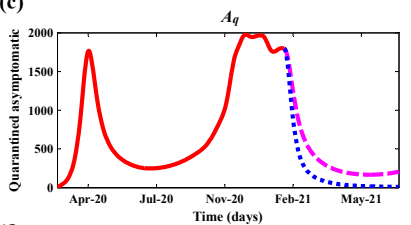

(f)

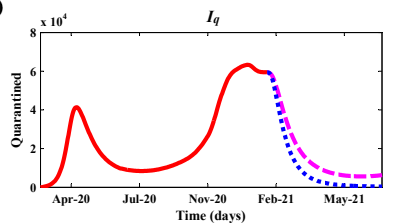

Figure 6. Projections for change in isolated class. Model simulations from 5 March 2020 to 18 July 2021 for (a) susceptible class, (b) asymptomatic class, (c) quarantined asymptomatic class, (d) isolated class, (e) infected class, (f) quarantined class, (g) cumulative reported deaths and (h) cumulative reported cases. The solid curves (red) represent the base case whereas the dashed curves (magenta) represent the current projections following the pattern of base case and the dotted curves (blue) represent the projections upto 18 July 2021 due to $16 \%$ increase of isolation from the base case. The parameter values are given in Table 3 .

Isolation is another critical factor for this pandemic as our findings indicate that a $16 \%$ increase of isolation from the base case may help to reduce $4 \%$ of cumulative reported deaths and $10 \%$ of cumulative reported cases over a five months period. Together with other results shown in Section 4. it is understood that isolation has a dramatic effect on the pandemic. This result is consistent with ongoing scenario in many places [12. There have additional advantages of isolation as it can prevent other infections [9, 17].

Although isolation can mitigate the SARS-CoV-2 spread, but in reality, the number of isolated individuals should decrease over time due to the economy reopening or if the pandemic continues for a longer period (as it happened worldwide during the current pandemic situation) [33. Consequently, isolated individuals may have to return to the susceptible pool in course of time which will possibly result in a massive increase of SARS-CoV-2. In order to curb such spread, a reasonable and oversized isolation policy is required 21 but this is challenging during economy reopening. In this scenario, the screening program could play a significant role [4, 6] as it detects the asymptomatic cases who are likely to transmit infections to others unknowingly. We suggest for an universal screening program if isolation can not be maintained.

In contrast to the positive results, there is a possibility of occurring a third wave of infection if isolation or screening is reduced to a certain extent. The model predicts that a reduction of $80 \%$ screening or $15 \%$ isolation from the base case may cause a resurgence of infection before 18 July 2021 . Due to the third wave, $11 \%$ to $12 \%$ of the total population of New Jersey is likely to get infected by the end of July 2021 along with 27.3 thousand deaths. There may be additional waves too. So, 
(a)

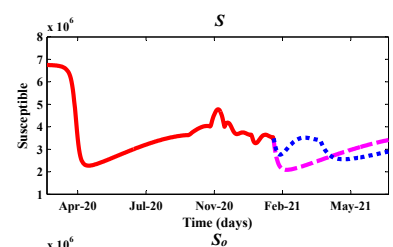

(d)

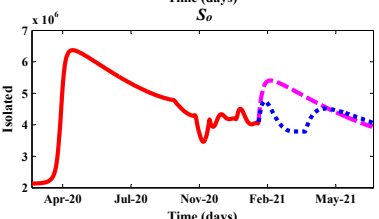

(g)

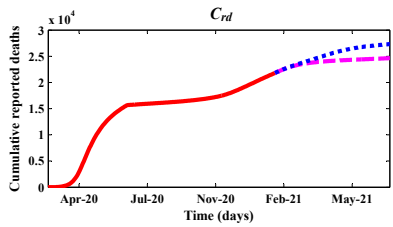

(b)

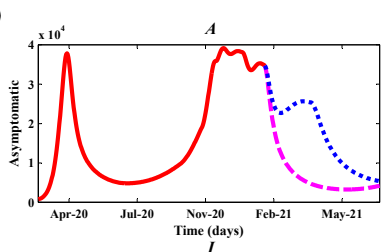

(e)

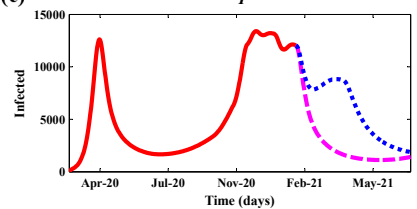

(h)

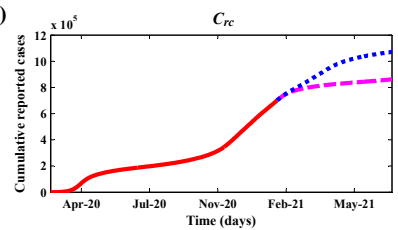

(c)

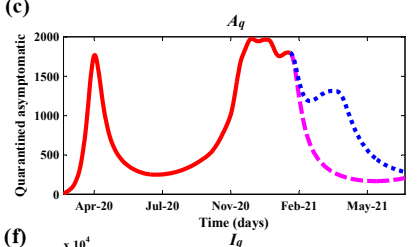

(f)
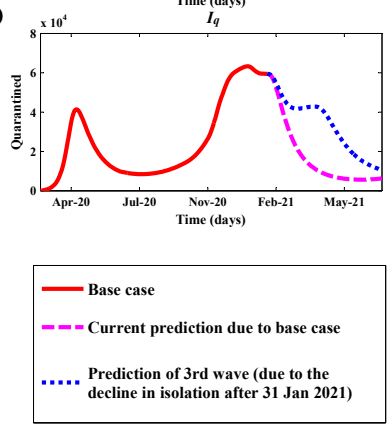

Figure 7. Prediction of 3rd wave due to the decline in isolated class. Model simulations from 5 March 2020 to 18 July 2021 for (a) susceptible class, (b) asymptomatic class, (c) quarantined asymptomatic class, (d) isolated class, (e) infected class, (f) quarantined class, (g) cumulative reported deaths and (h) cumulative reported cases. The solid curves (red) represent the base case whereas the dashed curves (magenta) represent the current projections following the pattern of base case and the dotted curves (blue) represent the projections upto 18 July 2021 due to $15 \%$ decline in isolation from the base case. The parameter values are given in Table 3.

it's important to know the approximate size of the infected population at the end of the pandemic. Therefore, the final size of our model, in that case, is an important threshold for the next possible prevention measurements.

Although our current model captures some important aspects of SARS-CoV-2 dynamics under screening program and dynamic isolation, it has limitations. First, our model does not incorporate any immigration. Since New Jersey is an open state, the individuals of New Jersey might come contact with the asymptomatic individuals of other states. As a result, there may be some variations in the results. Second, the model does not address vaccination. However, a fraction (subject to efficacy) of the vaccinated individuals can be considered as isolated and our results can be reinterpreted in accordance.

This study shows that dynamic isolation and screening program have been and will remain critical factors during this pandemic. While uncertainties remain in our results due to data gap and nonhomogeneity in population and their behaviour, the findings could be useful for a general understanding of the sensitivity of these interventions against the spread of SARS-CoV-2 or any upcoming pathogens. 
(a)

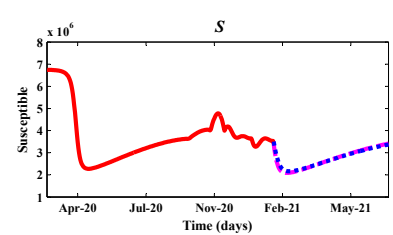

(d)

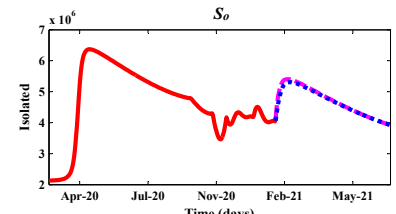

(g)

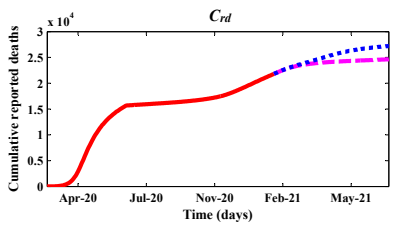

(b)

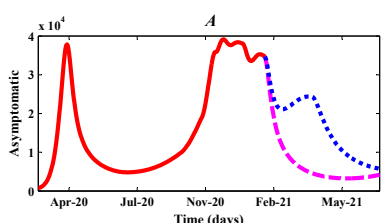

I

(e)

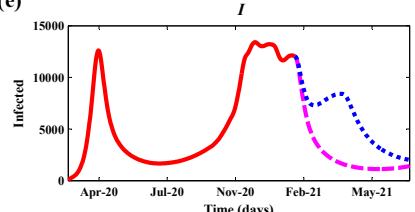

$C_{r c}$

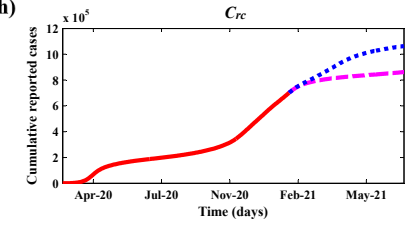

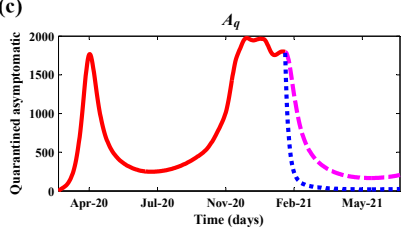

(f)
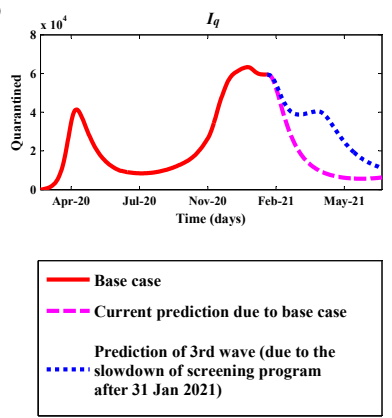

Figure 8. Prediction of 3rd wave due to the slowdown of screening program. Model simulations from 5 March 2020 to 18 July 2021 for (a) susceptible class, (b) asymptomatic class, (c) quarantined asymptomatic class, (d) isolated class, (e) infected class, (f) quarantined class, (g) cumulative reported deaths and (h) cumulative reported cases. The solid curves (red) represent the base case whereas the dashed curves (magenta) represent the current projections following the pattern of base case and the dotted curves (blue) represent the projections upto 18 July 2021 due to $80 \%$ reduction of screening program from the base case. The parameter values are given in Table 3 .

\section{REFERENCES}

[1] M. A. Acuña-Zegarra, M. Santana-Cibrian and J. X. Velasco-Hernandez, Modeling behavioral change and COVID-19 containment in Mexico: A trade-off between lockdown and compliance, Mathematical Biosciences 325(2020): 108370. https://doi.org/10.1016/j.mbs.2020.108370

[2] A. Aleta, D. Martín-Corral, A. P. y Piontti, M. Ajelli, M. Litvinova, M. Chinazzi, N. E. Dean, M. E. Halloran, I. M. L. Jr, S. Merler, A. Pentland, A. Vespignani, E. Moro and Y. Moreno, Modelling the impact of testing, contact tracing and household quarantine on second waves of COVID-19, Nature Human Behaviour 4(2020), 964971. https://doi.org/10.1038/s41562-020-0931-9

[3] J. Arino, F. Brauer, P. van den Driessche, J. Watmough and J. Wu, A final size relation for epidemic models, Mathematical Biosciences and Engineering 4(2007), 159-175. https://doi.org/10.3934/mbe.2007.4.159

[4] A. Asgary, M. G. Cojocaru, M. M. Najafabadi and J. Wu, Simulating preventative testing of SARS-CoV-2 in schools: policy implications, BMC Public Health 21(2021): 125. https://doi.org/10.1186/s12889-020-10153-1

[5] Y. Bai, L. Yao, T. Wei, F. Tian, D. Jin, L. Chen and M. Wang, Presumed Asymptomatic Carrier Transmission of COVID-19, JAMA 323(2020), 1406-1407. https://doi.org/10.1001/jama.2020.2565

[6] D. Berger, K. Herkenhoff, C. Huang and S. Mongey, Testing and reopening in an SEIR model, Review of Economic Dynamics (2020). https://doi.org/10.1016/j.red.2020.11.003

[7] Centers for Disease Control and Prevention, https://www.cdc.gov/coronavirus/2019-ncov/your-health/index. html, accessed January 31, 2021.

[8] N. Chitnis, J. M. Hyman and J. M. Cushing, Determining Important Parameters in the Spread of Malaria Through the Sensitivity Analysis of a Mathematical Model, Bulletin of Mathematical Biology 70(2008): 1272. https://doi. org/10.1007/s11538-008-9299-0

[9] Y. J. Choe and J. Lee, The Impact of Social Distancing on the Transmission of Influenza Virus, South Korea, 2020, Osong Public Health and Research Perspectives 11(2020), 91-92. https://doi.org/10.24171/j.phrp.2020.11.3.07 
[10] Z. Feng, J. W. Glasser and A. N. Hill, On the benefits of flattening the curve: A perspective, Mathematical Biosciences 326(2020): 108389. https://doi.org/10.1016/j.mbs.2020.108389

[11] M. Ferranna, D. Cadarette and D. E. Bloom, COVID-19 Vaccine Allocation: Modeling Health Outcomes and Equity Implications of Alternative Strategies, Engineering, in press (2021). https://doi.org/10.1016/j.eng.2021.03.014

[12] T. Girum, K. Lentiro, M. Geremew, B. Migora and S. Shewamare, Global strategies and effectiveness for COVID-19 prevention through contact tracing, screening, quarantine, and isolation: a systematic review, Tropical Medicine and Health 48(2020): 91. https://doi.org/10.1186/s41182-020-00285-w

[13] Q. Griette, J. Demongeot and P. Magal, A robust phenomenological approach to investigate COVID-19 data for France, Mathematics in Applied Sciences and Engineering 2(2021), 149-160. https://doi.org/10.5206/mase/14031

[14] Johns Hopkins University, Non-Pharmaceutical Interventions, https://ncrc.jhsph.edu/ non-pharmaceutical-interventions/, accessed February 5, 2021.

[15] W. O. Kermack and A. G. McKendrick, A contribution to the mathematical theory of epidemics, Proceedings of the Royal Society A 115(1927), 700-721. https://doi.org/10.1098/rspa.1927.0118

[16] M. A. Khan and A. Atangana, Modeling the dynamics of novel coronavirus (2019- $n$ Cov) with fractional derivative, Alexandria Engineering Journal 59(2020), 2379-2389. https://doi.org/10.1016/j.aej.2020.02.033

[17] I. Kuitunen, M. Artama, L. Mäkelä, K. Backman, T. Heiskanen-Kosma and M. Renko, Effect of Social Distancing Due to the COVID-19 Pandemic on the Incidence of Viral Respiratory Tract Infections in Children in Finland During Early 2020, The Pediatric Infectious Disease Journal 39(2020), e423-e427. https://doi.org/10.1097/INF. 0000000000002845

[18] L. Lan, D. Xu, G. Ye, C. Xia, S. Wang, Y. Li and H. Xu, Positive RT-PCR Test Results in Patients Recovered From COVID-19, JAMA 323(2020), 1502-1503. https://doi.org/10.1001/jama.2020.2783

[19] A. Li, Y. Wang, P. Cong and X. Zou, Re-examination of the impact of some non-pharmaceutical interventions and media coverage on the COVID-19 outbreak in Wuhan, Infectious Disease Modelling 6(2021), 975-987. https: //doi.org/10.1016/j.idm.2021.07.001

[20] Q. Li, B. Tang, N. L. Bragazzi, Y. Xiao and J. Wu, Modeling the impact of mass influenza vaccination and public health interventions on COVID-19 epidemics with limited detection capability, Mathematical Biosciences 325(2020): 108378. https://doi.org/10.1016/j.mbs.2020.108378

[21] M. Liu, R. Thomadsen and S. Yao, Forecasting the spread of COVID-19 under different reopening strategies, Scientific Reports 10(2020): 20367. https://doi.org/10.1038/s41598-020-77292-8

[22] M. Mandal, S. Jana, S. K. Nandi, A. Khatua, S. Adak and T. K. Kar, A model based study on the dynamics of COVID-19: Prediction and control, Chaos, Solitons \& Fractals 136(2020): 109889. https://doi.org/10.1016/j. Chaos.2020.109889

[23] Z. McCarthy, Y. Xiao, F. Scarabel, B. Tang, N. L. Bragazzi, K. Nah, J. M. Heffernan, A. Asgary, V. K. Murty, N. H. Ogden and $\mathrm{J} . \mathrm{Wu}$, Quantifying the shift in social contact patterns in response to non-pharmaceutical interventions, Journal of Mathematics in Industry 10(2020): 28. https://doi.org/10.1186/s13362-020-00096-y

[24] K. Nah, M. Alavinejad, A. Rahman, J. M. Heffernan and J. Wu, Impact of influenza vaccine-modified infectivity on attack rate, case fatality ratio and mortality, Journal of Theoretical Biology 492(2020): 110190. https://doi.org/ $10.1016 / j \cdot j$ tbi.2020.110190

[25] F. Ndairou, I. Area, J. J. Nieto and D. F. M. Torres, Mathematical modeling of COVID-19 transmission dynamics with a case study of Wuhan, Chaos, Solitons \& Fractals 135(2020): 109846. https://doi.org/10.1016/j.chaos. 2020.109846

[26] C. N. Ngonghala, E. Iboi, S. Eikenberry, M. Scotch, C. R. MacIntyre, M. H. Bonds and A. B. Gumel, Mathematical assessment of the impact of non-pharmaceutical interventions on curtailing the 2019 novel Coronavirus, Mathematical Biosciences 325(2020): 108364. https://doi.org/10.1016/j.mbs.2020.108364

[27] Q. Pan, S. Song and M. He, The effect of quarantine measures for close contacts on the transmission of emerging infectious diseases with infectivity in incubation period, Physica A: Statistical Mechanics and its Applications 574(2021): 125993. https://doi.org/10.1016/j.physa.2021.125993

[28] S. M. A. Rahman, N. K. Vaidya and X. Zou, Impact of early treatment programs on HIV epidemics: An immunitybased mathematical model, Mathematical Biosciences 280(2016), 38-49. https://doi.org/10.1016/j.mbs.2016.07. 009

[29] S. M. A. Rahman and X. Zou, Modelling the impact of vaccination on infectious diseases dynamics, Journal of Biological Dynamics 9(2015), 307-320. https://doi.org/10.1080/17513758.2014.986545

[30] A. M. Ramos, M. Vela-Pérez, M. R. Ferrández, A. B. Kubik and B. Ivorra, Modeling the impact of SARS-CoV-2 variants and vaccines on the spread of COVID-19, Communications in Nonlinear Science and Numerical Simulation 102(2021): 105937. https://doi.org/10.1016/j.cnsns.2021.105937 
[31] X. Rong, L. Yang, H. Chu and M. Fan, Effect of delay in diagnosis on transmission of COVID-19, Mathematical Biosciences and Engineering 17(2020), 2725-2740. https://doi.org/10.3934/mbe.2020149

[32] S. S. Shanta and M. H. A. Biswas, The Impact of Media Awareness in Controlling the Spread of Infectious Diseases in Terms of SIR Model, Mathematical Modelling of Engineering Problems 7(2020), 368-376. https://doi.org/10. $18280 / \mathrm{mmep} .070306$

[33] J. H. Stock, Reopening the Coronavirus-Closed Economy, Hutchins Center (2020).

[34] B. Tang, X. Wang, Q. Li, N. L. Bragazzi, S. Tang, Y. Xiao and J. Wu, Estimation of the Transmission Risk of the 2019-nCoV and Its Implication for Public Health Interventions, Journal of Clinical Medicine 9(2020): 462. https://doi.org/10.3390/jcm9020462

[35] J. Tian, J. Wu, Y. Bao, X. Weng, L. Shi, B. Liu, X. Yu, L. Qi and Z. Liu, Modeling analysis of COVID-19 based on morbidity data in Anhui, China, Mathematical Biosciences and Engineering 17(2020), 2842-2852. https: //doi.org/10.3934/mbe.2020158

[36] United States Census Bureau, QuickFacts New Jersey; United States, https://www.census.gov/quickfacts/fact/ table/NJ, US/PST045219, accessed January 31, 2021.

[37] P. van den Driessche and J. Watmough, Reproduction numbers and sub-threshold endemic equilibria for compartmental models of disease transmission, Mathematical Biosciences 180(2002), 29-48. https://doi.org/10.1016/ S0025-5564(02)00108-6

[38] D. Wang, B. Hu, C. Hu, F. Zhu, X. Liu, J. Zhang, B. Wang, H. Xiang, Z. Cheng, Y. Xiong, Y. Zhao, Y. Li, X. Wang and Z. Peng, Clinical Characteristics of 138 Hospitalized Patients With 2019 Novel Coronavirus-Infected Pneumonia in Wuhan, China, JAMA 323(2020), 1061-1069. https://doi.org/10.1001/jama.2020.1585

[39] L. Wang, J. Wang, H. Zhao, Y. Shi, K. Wang, P. Wu and L. Shi, Modelling and assessing the effects of medical resources on transmission of novel coronavirus (COVID-19) in Wuhan, China, Mathematical Biosciences and Engineering 17(2020), 2936-2949. https://doi.org/10.3934/mbe.2020165

[40] X. Wang, Q. Li, X. Sun, S. He, F. Xia, P. Song, Y. Shao, J. Wu, R. A. Cheke, S. Tang and Y. Xiao, Effects of medical resource capacities and intensities of public mitigation measures on outcomes of COVID-19 outbreaks, BMC Public Health 21(2021): 605. https://doi.org/10.1186/s12889-021-10657-4

[41] G. Webb, A COVID-19 epidemic model predicting the effectiveness of vaccination, Mathematics in Applied Sciences and Engineering 2(2021), 134-148. https://doi.org/10.5206/mase/13889

[42] Worldometer, Coronavirus Updates in New Jersey, https://www.worldometers.info/coronavirus/usa/ new-jersey/, accessed January 31, 2021.

[43] J. T. Wu, K. Leung and G. M. Leung, Nowcasting and forecasting the potential domestic and international spread of the 2019-nCoV outbreak originating in Wuhan, China: a modelling study, The Lancet 395(2020), 689-697. https://doi.org/10.1016/S0140-6736(20)30260-9

[44] C. Yang and J. Wang, A mathematical model for the novel coronavirus epidemic in Wuhan, China, Mathematical Biosciences and Engineering 17(2020), 2708-2724. https://doi.org/10.3934/mbe.2020148

[45] W. Zhou, A. Wang, F. Xia, Y. Xiao and S. Tang, Effects of media reporting on mitigating spread of COVID-19 in the early phase of the outbreak, Mathematical Biosciences and Engineering 17(2020), 2693-2707. https://doi.org/ $10.3934 / \mathrm{mbe} .2020147$

Department of Mathematics and Natural Sciences, Brac University, Dhaka 1212, Bangladesh

E-mail address: azmir.islam@bracu.ac.bd

Mathematics Discipline, Khulna University, Khulna 9208, Bangladesh

E-mail address: ss_shanta@math.ku.ac.bd

Corresponding author, Department of Mathematics and Statistics, Oakland University, Rochester, Mi 48309

E-mail address: rahman2@oakland.edu 